\title{
Design of Dispersion Section for the ATF Harmonic Generation FEL *
}

\author{
X. Zhang, I. Ben-Zvi, G. Ingold, S. Krinsky, and L. H. Yu \\ National Synchrotron Light Source \\ Brookhaven National Laboratory \\ Upton, NY 11973
}

\begin{abstract}
We present an analysis of the dispersion magnet for the high gain harmonic generation experiment to be carried out at the Accelerator Test Facility at BNL. The purpose of the dispersion magnet is to convert energy modulation of the electron beam into spatial bunching. For the dispersion magnet, we discuss the physical requirements, analyze the magnetic design, determine the focussing properties, and consider the effect on the FEL gain of departures from ideal behavior on the FEL gain.
\end{abstract}

\section{Introduction}

The High Gain Harmonic Generation experiment (HGHG) [1] at the Accelerator Test Facility (ATF) at BNL provides a proof of principle for the design of a proposed UV. FEL User Facility $[2,3]$. In the experiment it is planned to triple the frequency of a $\mathrm{CO}_{2}$ seed laser by utilizing two superconducting wigglers separated by a dispersion section as shown in Fig. 1. The first wiggler, resonant to $10.4 \mu \mathrm{m}$, is used to modulate the energy of the electron beam through the ponderomotive force resulting from the $\mathrm{CO}_{2}$ seed laser. In the dispersion section, the particles with higher energy will be deflected less effectively and thus travel a short path length than those particles with lower energy. As a result, the electrons are bunched. This coherent spatially bunched beam induces superradiant emission at the third harmonic when it passes through the second wiggler, which is tuned to resonate at this harmonic. Characteristically, along the second wiggler the radiation initially grows quadratically, then exponentially in untapered section and quadratically again in tapered section after saturation.

-This work was performed under the auspices of the U.S. Department of Energy. 


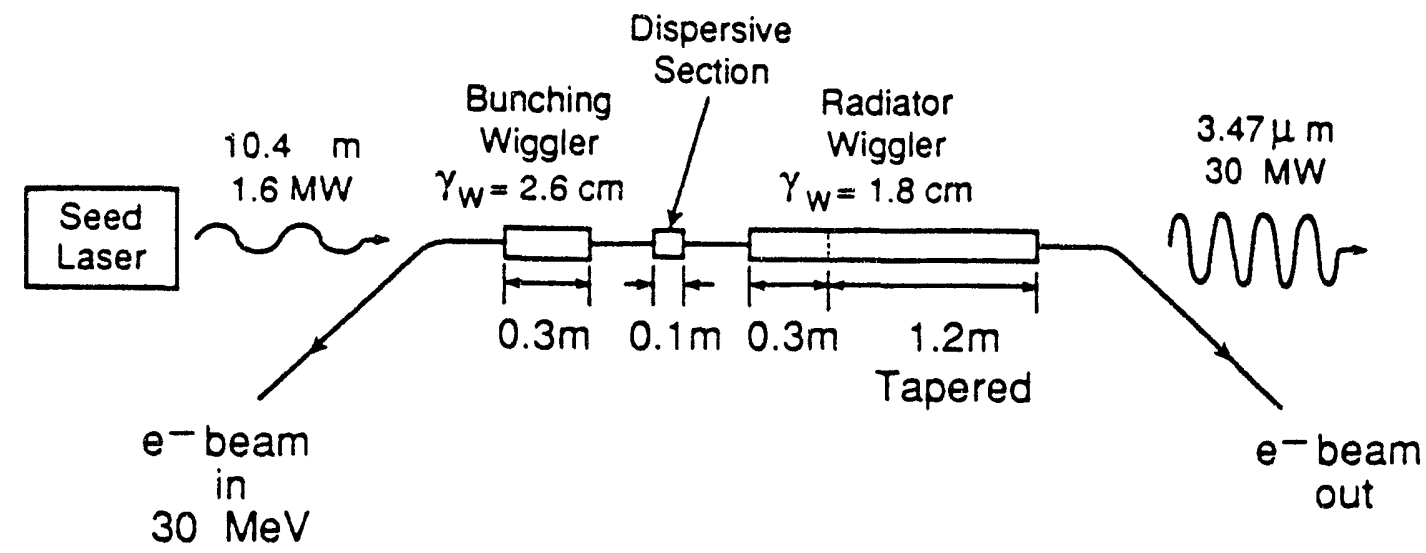

Figure 1: Schematic of the ATF HGHG experiment.

The design of the dispersion section and its modelling for the HGHG experiment is presented in this paper. The requirements on the dispersion section are discussed in section II. A detailed design of the dispersion magnet is presented in section III. In section IV, the electron beam optics in the designed magnet is modelled and its effects on the FEL are examined.

\section{Requirements on Dispersion Section}

The dispersion section is used to convert an energy modulation produced in the first wiggler into a spatial bunching. The ideal dispersion section is an achromat in which the longitudinal phases $\left[\psi \equiv\left(k_{s}+k_{w}\right) z-\omega_{s} t\right]$ of a particle changes in proportion to the deviation from the average energy,

$$
\Delta \psi=\frac{d \psi}{d \gamma} \Delta \gamma
$$

We assume a two-dimensional magnetic field whose vertical component $B$ is expressed in terms of the horizontal component of the vector potential $\mathrm{A}$ by $B=\partial A / \partial z$. The path length $s$ in the mid-plane can be written in terms of particle momentum $p$ and vector potential $A[4]$

$$
s(z)=\int_{-\infty}^{z} \frac{a_{0}}{\sqrt{a_{0}^{2}-A\left(z^{\prime}\right)^{2}}} d z^{\prime},
$$

where $a_{0}=p / e$. Using the paraxial and highly relativistic approximations, the dispersion $d \psi / d \gamma$ can be written as a function of the vertical magnetic field in the magnet [5]

$$
\frac{d \psi}{d \gamma}=k_{s} \frac{d s}{d \gamma}=\frac{e^{2} k_{s}}{m^{2} c^{2} \gamma^{3}} \int_{0}^{L_{d}} d z\left[\int_{0}^{z} d z^{\prime} B\left(z^{\prime}\right)\right]^{2},
$$

where $k_{s}$ is the resonant wavenumber of the second wiggler, $\gamma$ is the electron beam energy, and $L_{d}$ is the length of the dispersion section. The desired value of the 
dispersion is set by optimization of the output radiation power. Additional conditions on the dispersion section are that the first and second integrals of the magnetic field over the distance $L_{d}$ should vanish so that the beam will have no position and angular displacement resulting from the dispersion section. One example of an idealized dispersion section has magnetic field given by

$$
B(z)=\left\{\begin{aligned}
B_{0}, & 0 \leq z<\frac{L_{d}}{4} \\
-B_{0}, & \frac{L_{d}}{4}<z<\frac{3 L_{d}}{4} \\
B_{0}, & \frac{3 L_{d}}{4}<z<L_{d}
\end{aligned}\right.
$$

This field can be obtained by a system consisting of 4 identical rectangular magnets (the field in $L_{d} / 4 \sim 3 L_{d} / 4$ provided by two dipoles). Obviously, this configuration satisfies the two integral conditions. The required dispersion can be achieved by adjusting the parameters $B_{0}$ and $L_{d}$. Substituting the field of Eq. (4) in Eq. (3), the dispersion is found to be

$$
\frac{d \psi}{d \gamma}=\frac{e^{2} k_{s}}{m^{2} c^{2} \gamma^{3}} \frac{B_{0}^{2} L_{d}^{3}}{48},
$$

which shows that the dispersion is proportional to $B_{0}^{2}$ and $L_{d}^{3}$ and inversely proportional to $\gamma^{3}$. The maximum horizontal deflection $x_{\max }$ in the dispersion section is another parameter important in design. Due to the symmetry, the $x_{\max }$ is reached at the center $\left(L_{d} / 2\right)$ of the dispersion section. This maximum value in the mid-plane is given by

$$
x_{\max }=2 \rho(1-\cos \theta),
$$

where $\rho$ is bending radius in a rectangular magnet of length $L_{d} / 4$ and

$$
\sin \theta=\frac{L_{d}}{4 \rho}
$$

For $L_{d} / 4 \rho \ll 1,1-\cos \theta \approx \theta^{2} / 2$ and $\theta \sim \sin \theta \approx L_{d} / 4 \rho$, then

$$
x_{\text {max }} \approx \rho \theta^{2} \approx \frac{B_{0} L_{d}^{2}}{16 m c \gamma} \text {. }
$$

Under this assumption of small bending, it is seen that the maximum deflection is inversely proportional to the energy of the particles.

The related system parameters in the HGHG experiment, currently under construction, are listed Table 1 . In order to minimize the wakefield effects, we choose the gap of the dispersion section to be the same as that of the other two wigglers. The horizontal pole width should be enough to keep constant field to the maximum deflection. From Eqs. (5) and (6), for a fixed dispersion $d \psi / d \gamma$ and a given electron energy $\gamma$, the choice of higher magnetic field with shorter length of dispersion section minimizes $x_{\text {max }} e$. The length of dispersion section in the HGHG experiment is $10 \mathrm{~cm}$. 
Table 1: System Parameters [(1) and (2) stand for first wiggler and second wiggler respectively].

\begin{tabular}{|l|c|}
\hline Energy, $[\mathrm{MeV}]$ & 30 \\
\hline Peak Current, $[\mathrm{Amp}]$ & 110 \\
\hline Normalized RMS Emittance,[mm-mrad] & 4.0 \\
\hline RMS Energy Spread [\%] & 0.0435 \\
\hline e-beam Edge Radius $(1)[\mathrm{mm}]$ & 0.424 \\
\hline e-beam Edge Radius $(2)[\mathrm{mm}]$ & 0.525 \\
\hline Resonant Wavelength $(1)[\mu \mathrm{m}]$ & 10.42 \\
\hline Betatron Wavelength $(1)[\mathrm{m}]$ & 1.608 \\
\hline Wiggler period $(1)[\mathrm{mm}]$ & 26 \\
\hline Resonant Wavelength $(2)[\mu \mathrm{m}]$ & 3.47 \\
\hline Betatron Wavelength $(2)[\mathrm{m}]$ & 2.466 \\
\hline Wiggler period $(2)[\mathrm{mm}]$ & 18 \\
\hline Wiggler gap $(1,2)[\mathrm{mm}]$ & 8 \\
\hline
\end{tabular}

\section{Magnetic Design and Results}

We have studied the dispersion magnet design using the 2-D computer code POISSON. Our dispersion section is a single period electromagnetic wiggler with a uniform gap. The right-upper quarter of the dispersion magnet is shown in Fig. 2, The rest is determined by the symmetry of the system. The boundary conditions are Dirichlet on the left boundary and Neumann on the right, upper and lower boundaries.

Following a suggestion of $\mathrm{K}$. Halbach [4], we use field clamps at the ends of the dispersion section to reduce the fringe fields leaking out from the magnet ends. Thus there are five iron poles, one long main pole, two side poles and two end poles. Three coil with equal current are wound around the three magnetic field poles. the ratio of the side pole to main pole is slightly different from $1: 2: 1$ to make the first field integral vanish over the half dispersion section. The second integral vanishes automatically by symmetry.

The field clamp provides a low reluctance connection between the upper and lower end poles, placing them at scalar potential equal to that of the mid-plane. Window-frame type field clamp shown in Fig. 3 are connected at the both ends of the magnets. To simulate the 3-dimensional field clamp using the 2-dimensional program POISSON, we use a procedure developed by Halbach [4]. A fictitious iron bar with infinite permeability is used to connect the outer edge of the dispersion magnet to the right boundary of the calculated region, as shown in Fig. 4. This makes integral $\int \vec{H} \cdot \overrightarrow{d l}$ from the magnet end to the mid-plane vanish, since the right end of the problem has a Neumann boundary condition. This places the end pole at 


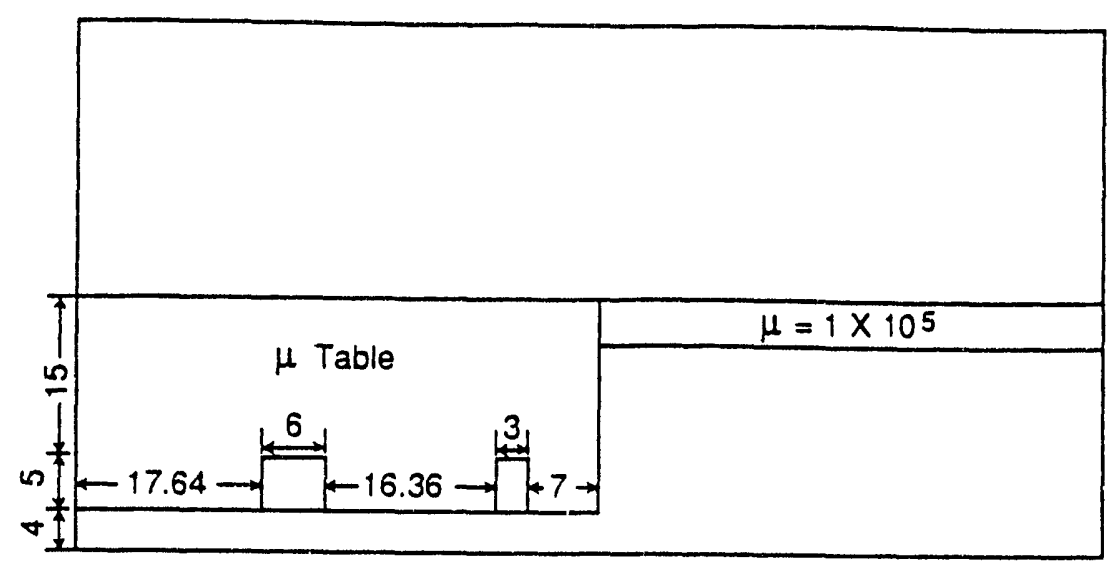

Figure 2: Detailed description of right-upper quarter of the dispersion magnet with virtual feld clamp, showing dimension in $\mathrm{mm}$ and permeability.

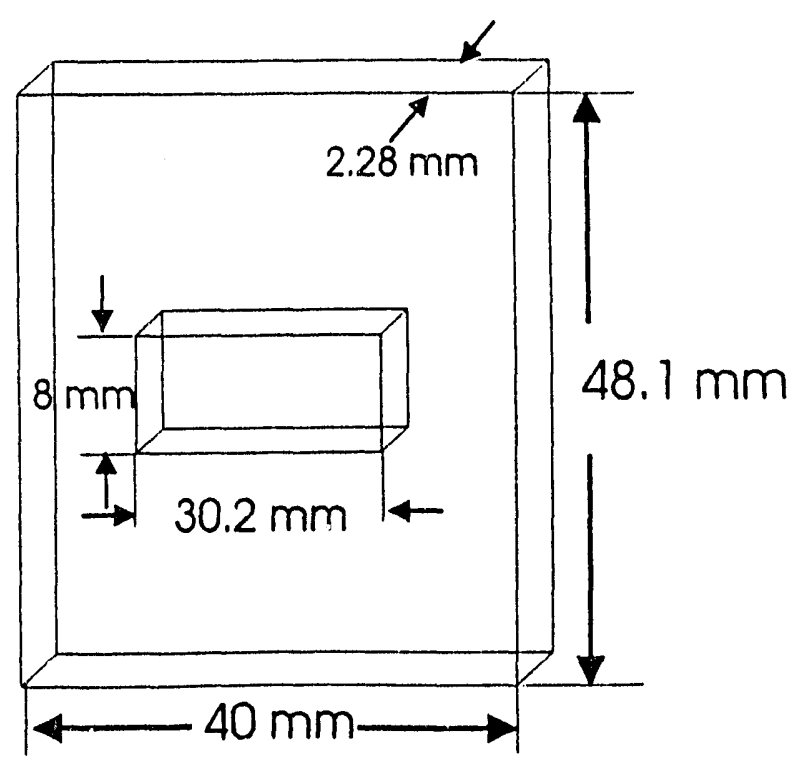

Figure 3: Field clamp at the end of the magnet. 


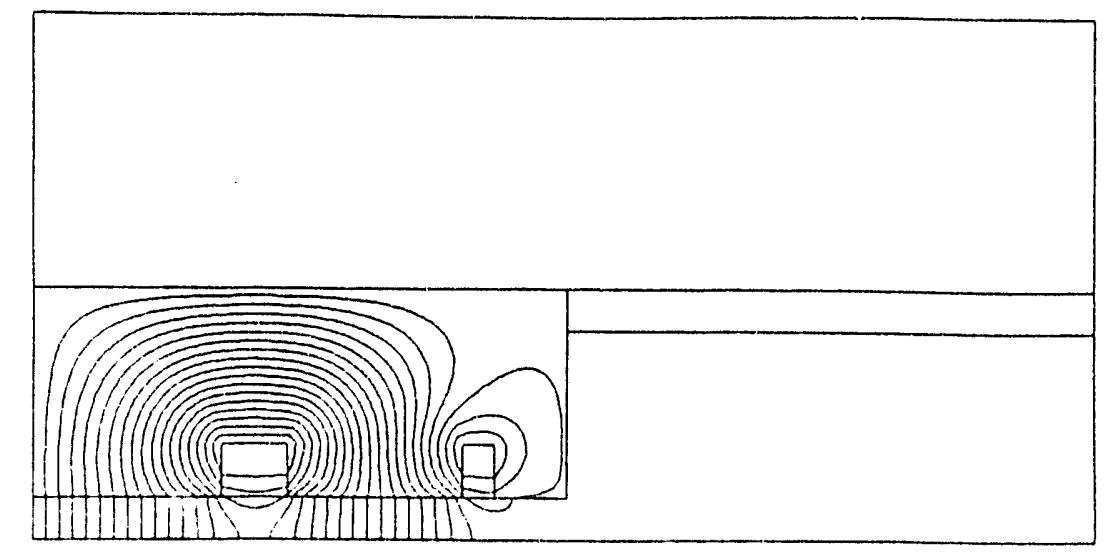

Figure 4: POISSON field line plots of dispersion magnet.

equal scalar potential to the mid-plane. In the regime of low saturation, the field in the body of the magnet is only slightly modified by the field clamp, since little flux passes through the clamp.

The field line plot of the dispersion wiggler is shown in Fig. 4. In this calculation, we use the variable permeability table for wiggler iron and large $\left(\mu=1 \times 10^{5}\right)$, constant permeability for the field clamp bar. The coils are excited with $2800: 5600$ Ampereturns to provide $d \psi / d \gamma=22$ required by the HGHG experiment. Note that there are no field lines inside the simulated field clamp. The field in the mid-plane of the hali magnet is shown in Fig. 5. The field amplitude at the central pole is approximately equal to that at the side pole, but the polarity of the field is inverted. The field vanishes quickly in the field clamp pole.

The field in the mid-plane and the dispersion are displayed as a function of the coil excitation in Fig. 6. With the excited current within 5000 Ampere-turns, the magnet generates 1.4 Tesla peak field and a dispersion of 50, while operating almost in the linear region. The first field integral in the half magnet which is nearly zero for the nominal current, is about 60 Gauss $-\mathrm{cm}$ at an excitation of 5000 Ampere-turns. This corresponds to $0.6 \mathrm{mrad}$ beam steering. Since this value is twice larger than the tolerance required by the experiment, a pair of small trim coils would be needed if dispersion has to be adjusted to such an extreme value.

The coils are made of superconducting wire with diameter of $0.44 \mathrm{~mm}$. The main coils are twice the size of the side coils, which have area $5 \times 3 \mathrm{~mm}^{2}$. The number of turns in the side coil is 63 . The excited current per turn is below 80 Ampere, well below the short sample quench current, which is over 200 Amperes.

This dispersion magnet can also be used in the proposed NSLS UV-FEL, where the typical radiation wavelength is $100 \mathrm{~nm}$ and electron beam energy is $260 \mathrm{MeV}$. With $6 \mathrm{~mm}$ gap and 2800:5600 Ampare-turns coil excitation, the magnet can generate field with peak value $1.156 \mathrm{~T}$ and produce the dispersion of 2.5 needed for that device. 


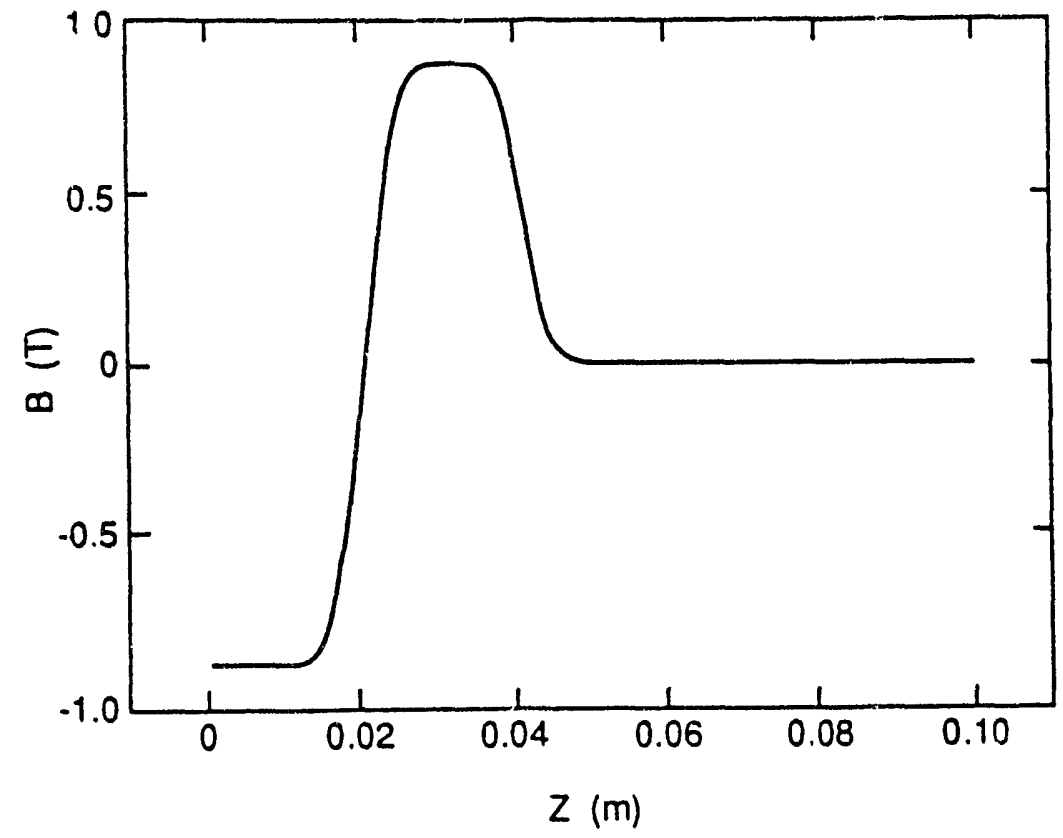

Figure 5: Mid-plane field vs longitudinal distance of dispersion magnet.

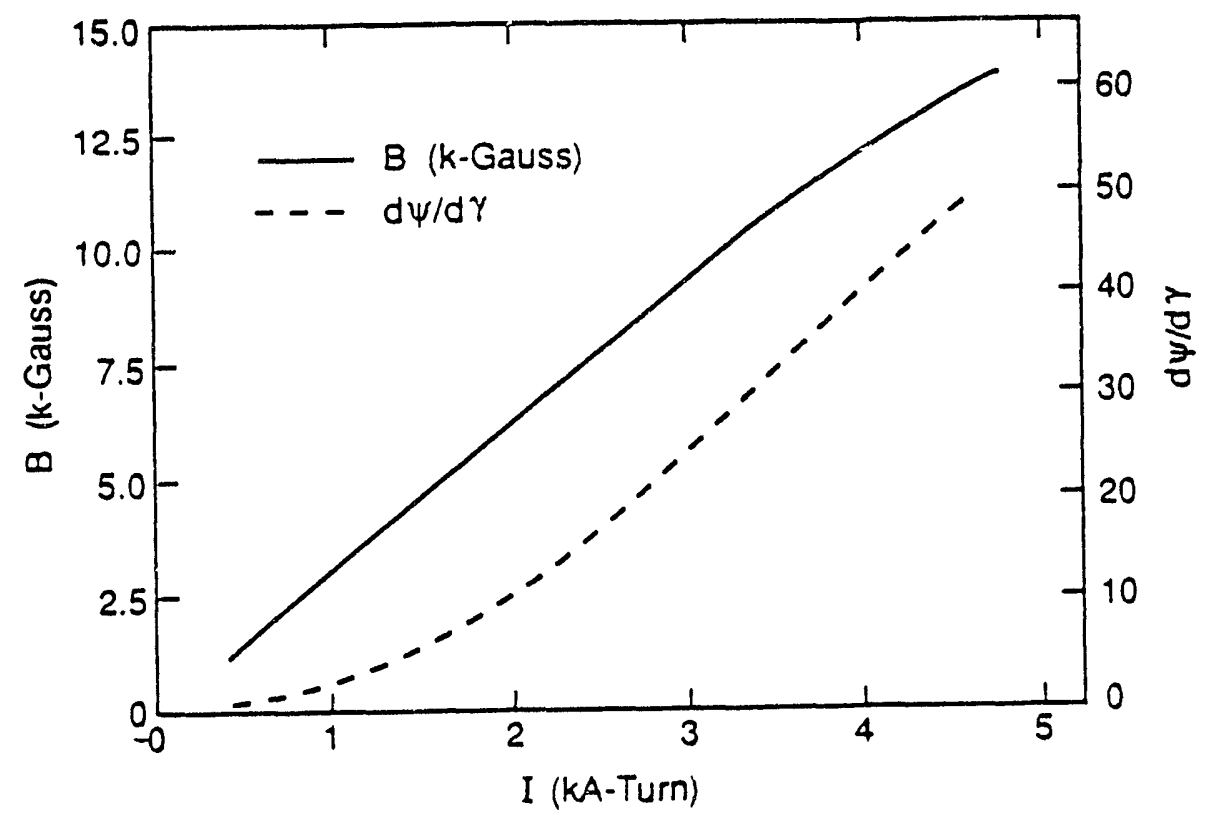

Figure 6: Mid-plane field and dispersion vs current. 


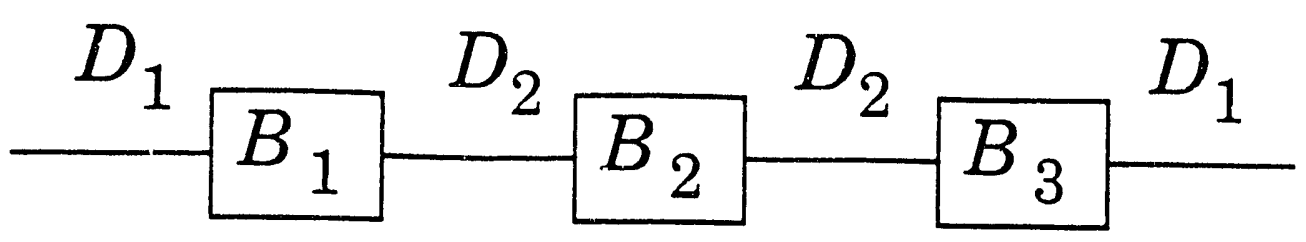

Figure 7: Modelled beam optics for dispersion section.

\section{Modelling of Dispersion Section}

The dispersion section is designed with no horizontal focussing. As a result, the beam can only be matched to one of the two wigglers. This effect may cause some reduction of the FEL radiative power. To estimate this effect, we have to determine the transport matrix of the dispersion section.

For this purpose, the dispersion wiggler is modelled by a collection of dipole magnets and drifts. Since the first and second field integrals are equal to zero, we use three rectangular dipoles (B1, B2 and B3) to form an achromatic bending device as shown in Fig. 7. The length of the dipole magnet is adjusted to make the field integral over each rectangular pole equal to the integral found by POISSON for the true geometry. The drifts make up extra space. The parameters of these elements shown in Fig. 7 are listed in Table 2. Since the field between the poles does not in reality show a step funciion-like jump, the hard edge model of dipole magnet overestimates the focussing effects in vertical plane due to the fringe field. Therefore, we employ a correction term taking into account spatial extent of fringing fields [7], which is related to $K_{1}$ given by

$$
K_{1}=\int_{-\infty}^{\infty} \frac{B(z)\left(B_{0}-B(z)\right)}{g B_{0}^{2}} d z
$$

where $B(z)$ is the magnitude of the fringing field on the mid-plane at a position $z$, $B_{0}$ is the asymptotic value of $B(z)$ inside the magnet, and $g$ is full gap of bending magnet. The values of $K_{1}$ are given in Table 2.

The transport matrix shown in Eq. (9) was produced by TRANSPORT code, which includes the drift space between the dispersion section and two wigglers

$$
R=\left(\begin{array}{rrrrrr}
1.00002 & 0.18074 & 0.00000 & 0.00000 & 0.00000 & 0.00000 \\
0.00025 & 1.00002 & 0.00000 & 0.00000 & 0.00000 & 0.00000 \\
0.00000 & 0.00000 & 0.67546 & 0.15184 & 0.00000 & 0.00000 \\
0.00000 & 0.00000 & -3.58112 & 0.67546 & 0.00000 & 0.00000 \\
0.00000 & 0.00000 & 0.00000 & 0.00000 & 1.00000 & 0.00739 \\
0.00000 & 0.00000 & 0.00000 & 0.00000 & 0.00000 & 1.00000
\end{array}\right)
$$


Table 2: Parameters of the modelled dispersion section.

\begin{tabular}{|l|c|c|c|c|}
\hline Component & Length $(\mathrm{cm})$ & Field (T) & $K_{1}$ (in) & $K_{1}$ (out) \\
\hline D1 & 4.93 & & & \\
\hline D2 & 0.45 & & & \\
\hline B1 & 1.81 & 0.876 & 0.35 & 0.25 \\
\hline B2 & 3.62 & 0.876 & 0.25 & 0.25 \\
\hline B3 & 1.81 & 0.876 & 0.25 & 0.35 \\
\hline
\end{tabular}

This matrix shows that the dispersion section behaves nearly as a drift horizontally and as a thick focussing lens vertically. Converting the $R_{56}$ term (with unit $m m / \%$ ) in the matrix to the dispersion defined in Eq. (2), the result is in good agreement with the value calculated from the POISSON output. In addition, the maximum deflection occurring at the symmetric plane of the dispersion wiggler is found to be $3.4 \mathrm{~mm}$ in the case given by Table 2 , which is in agreement with the POISSON result. Using the model we can investigate second order effects on the longitudinal phase shift, such as the effects due to transverse position and angle of the beam. The results given by TRANSPORT show that second order effects are negligibly small.

In the HGHG experiment, the beam is matched to the second wiggler, but not to the first wiggler. Using the transport matrix of the dispersion section and the first wiggler, we can work out the required beam phase space parameters at the entrance of the first wiggler. To determine effect on the radiated output power, a simulation using code TDA [8] was employed with the calculated transport matrix and beam parameters. The result showed there was no significant reduction of the FEL radiated power due to the lack of matching into the first wiggler.

\section{ACKNOWLEDGMENTS}

We express our thanks to K. Halbach for his suggestions, discussions and comments. We also thank D. Li for his FEL computer simulation.

\section{DISCLAIMER}

This report was prepared as an account of work sponsored by an agency of the United States Government. Neither the United States Government nor any agency thereof, nor any of their employees, makes any warranty, express or implied, or assumes any legal liability or responsibility for the accuracy, completeness, or usefulness of any information, apparatus, product, or process disclosed, or represents that its use would not infringe privately owned rights. Reference herein to any specific commercial product, process, or service by trade name, trademark, manufacturer, or otherwise does not necessarily constitute or imply its endorsement, recommendation, or favoring by the United States Government or any agency thereof. The views and opinions of authors expressed herein do not necessarily state or reflect those of the United States Government or any agency thereof. 


\section{References}

[1] I. Ben-Zvi, et al., Proc. of 1991 Inte. FEL Conf., Santa Fe, NM.

[2] L. H. Yu, Physical Review A 44 5178(1991).

[3] I. Ben-Zvi, et al., to be appear in Nucl. Inst. \& Meth..

[4] K. Halbach, Private communication.

[5] R. Bonifacio, et al., Nucl. Inst. \& Meth. A296, 787(1990).

[6] K. Halbach, Nucl. Inst. \& Meth. A250 95(1986).

[7] K. Brown, SLAC Report SLAC-75 74(1982).

[8] T. M. Tran, Comput. Phys. Commun. 54, 263(1989). 

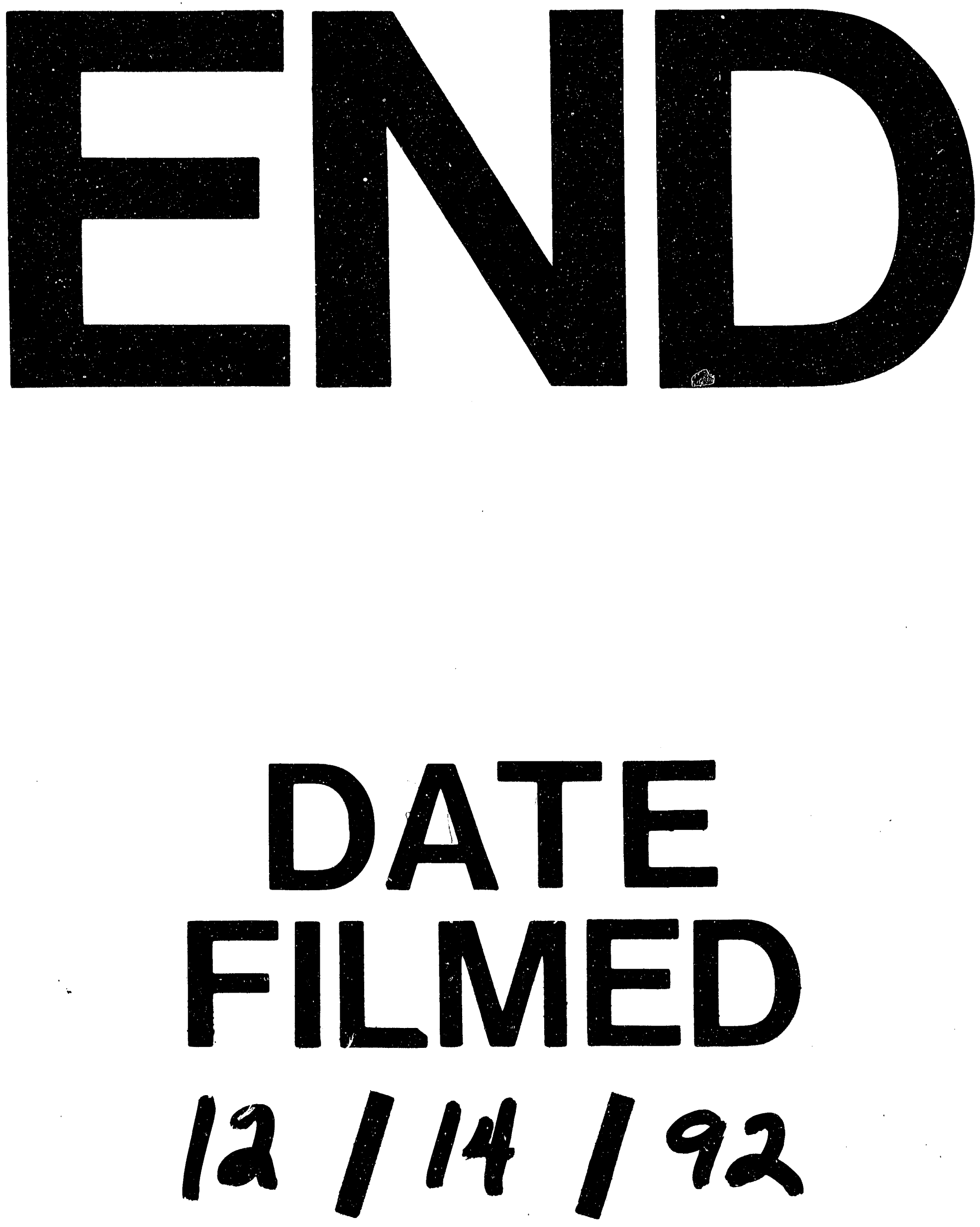
